\title{
USING WORKSTATION DESIGN PARAMETERS TO PREDICT WORKLOAD - A PROACTIVE ASSESSMENT APPROACH FOR LIGHT ASSEMBLY WORK
}

\author{
Michael A. Greig, \\ Human Factors Engineering Lab, Department of Mechanical and Industrial Engineering, \\ Ryerson University, 350 Victoria St. Toronto, Ontario, Canada, B5B 2K3. \\ m2greig@ryerson.ca, \\ Tim L. Annett, \\ Research in Motion Ltd., Ergonomics Group, Waterloo, Ontario, Canada, \\ Judy L. Village, W. Patrick Neumann, \\ Ryerson University, Toronto, Ontario, Canada
}

\begin{abstract}
Addressing the need for a virtual tool to predict operator load in light assembly work, a method is presented to estimate shoulder load and hand movement from layout parameters. Using three-dimensional representation of a task location relative to the seated workstation, a regression model is used to predict operator shoulder load. Hand locations for each task of the work cycle are used to determine cumulative hand movement and shoulder load. A case application of the virtual tool showed that trends from an observational tool used by Neumann et al. (2002) in a workstation comparison were matched. The virtual tool predicted shoulder load values $19.8-32.8 \%$ higher than the observational tool however this was attributed to its use of three-dimensional task analysis and a different shoulder model. Future work for the virtual tool will assess reliability, validity, the effect of underlying tool assumptions, and the incorporation of a movement time prediction method.
\end{abstract}

Keywords: virtual tool, shoulder load, workstation design

\section{UTILISATION DES PARAMĖTRES DE CONCEPTION DES POSTES DE TRAVAIL POUR PRÉDIRE LA CHARGE DE TRAVAIL - UNE APPROCHE D'ÉVALUATION PROACTIVE DU TRAVAIL D'ASSEMBLAGE LÉGER}

Pour examiner la nécessité d'un outil virtuel visant à prédire la charge demandée à un opérateur qui effectue un travail d'assemblage léger, nous présentons une méthode qui permet d'évaluer la charge à l'épaule et le mouvement de la main à partir de paramètres physiques. À l'aide d'une représentation tridimensionnelle de l'emplacement d'une tâche par rapport au poste de travail assis, un modèle de régression permet de prédire la charge demandée à l'épaule de l'opérateur. Les emplacements de la main pour chaque tâche du cycle de travail sont utilisés pour déterminer les mouvements cumulatifs de la main et de la charge à l'épaule. L'application d'un outil virtuel démontre que les tendances relevées sur un outil d'observation utilisé par Neumann et coll. (2002) dans la comparaison d'un poste de travail correspondent. L'outil virtuel prévoit des valeurs de charge à l'épaule 19,8-32,8\% plus élevées que l'outil d'observation, cependant ces résultats sont attribuables à l'usage d'une analyse de tâche tridimensionnelle et à un modèle d'épaule différent. D'autres travaux effectués sur l'outil virtuel permettront d'évaluer sa fiabilité, sa validité, les effets des hypothèses fondamentales de l'outil, et l'incorporation d'une méthode de prédiction de la durée du mouvement.

Mots clés : outil virtuel, charge à l'épaule, conception d'un poste de travail 


\section{$1.0 \quad$ INTRODUCTION}

Musculoskeletal injuries continue to be a concern for manufacturing workplaces. Most commonly, ergonomics has been used in response to worker injuries to assess task suitability, recommend changes and develop work accommodations reactively. Significant effort has been made in the ergonomics field to be more proactive in the prediction of operator risk exposure, which has been shown to be more cost effective (eg. Miles and Swift, 1998; Dul and Neumann, 2009). The development of tools that work in a 'virtual' workstation stage in the design process can help ergonomists work more proactively. Unfortunately ergonomists remain challenged to work upstream in part because there remain a limited number of tools available to work in this manner. A number of assessment tools exist that can be applied to large manufacturing and material handling (eg. RULA, NIOSH equations, Snook Tables, Energy Expenditure). Fewer tools are available for light assembly work such as in the electronics sector. While digital human models (eg. Jack, Michigan 3DSSPP, Watbak) are available and can help, these tools are expensive, and often rely on observation of posture, making them non-virtual. Otherwise they require a three-dimensional CAD representation of the workplace to be useful. Subsequently the cost of purchasing and having the technical staff to operate and interpret the models makes them financially prohibitive to most firms. What is required for light assembly work assessment is a virtual tool that is simple to use and easy to interpret. It must generate results quickly using only design parameters for input information and also be able to capture the effects of highly repetitive but low peak force task loads and the cumulative loading required for a full shift.

\section{$1.1 \quad$ AIM}

The aim of this paper is to introduce a simple, cost effective, virtual method to predict worker shoulder load in light assembly. The use of the tool will be demonstrated in a case example comparing a workstation re-design originally presented by Neuman et al (2002).

\subsection{METHODS}

\section{$2.1 \quad$ INPUTS}

Primary tool inputs in this method are the three-dimensional hand location coordinates for each task in a work cycle (eg. where the hand is located in space to pick up a part or place it on the workstation to work with it). Values for both the left and right hand are measured relative to an origin at the top of the workstation's surface at the front edge closest to the worker and centred in front of the body. The three-dimensional information can be estimated from design (CAD) drawings, known workstation configurations, or otherwise predicted from anticipated part locations when working in a virtual or design stage.

\subsection{SHOULDER LOAD REGRESSION MODEL}

Shoulder load regression models, both absolute load and as a percent of population maximum capabilities, were created using a two-dimensional biomechanical model (Watbak; University of Waterloo, Waterloo, ON). Regression equations were created based on the hand location coordinates relative to the shoulder for a series of elbow and shoulder flexion postures including $0,30,60,90^{\circ}$ for both segments. The positions covered the range of movement from the arm lowered at the side to a hand position not exceeding horizontal from the shoulder. "Light" assembly part weights were assumed to be negligible meaning no external hand forces were included in the analysis. Maximum capability was calculated from 
population maximums reported in the Watbak software for a given posture and used to calculate a percent of maximum voluntary contraction output for a second set of regression equations. Summary information for each of these models, in this proof of principle demonstration, for a $95^{\text {th }}$ percentile male and $50^{\text {th }}$ percentile female are shown in Table 1. The two percentiles were chosen based on prior population data used in earlier development work for a shoulder load regression model. The $50^{\text {th }}$ percentile female population also most closely matches the anthropometrics modelled by Neumann et al. (2002). All regression equations were statistically significant and R-Square values were higher for net shoulder moment compared to percent of capability for all models.

Table 1. Shoulder load regression equation values developed for two different populations and absolute or relative shoulder load. Where $\mathrm{x}$ is horizontal distance $(\mathrm{cm})$ and $\mathrm{y}$ is vertical distance $(\mathrm{cm})$ from the shoulder.

\begin{tabular}{lcccc}
\hline Data Set & R Square & Intercept & X (hand-shldr) & Y (hand-shldr) \\
\hline 95 \%ile Male (Nm) & 0.92 & -4.48 & 25.86 & -3.47 \\
50\%ile Female (Nm) & 0.91 & -2.31 & 14.71 & -2.06 \\
95 \%ile Male (\%MV) & 0.84 & -0.07 & 0.41 & -0.03 \\
50\%ile Female (\%MVC) & 0.85 & -0.08 & 0.50 & -0.06 \\
\hline
\end{tabular}

To apply the model, task hand locations were transposed from the origin to be relative to the shoulder joint to be compatible with the shoulder model. Standard shoulder to table origin distances were obtained from an available reference (Humanscale; Henry Dreyfuss and Associates, 1981). The three-dimensional position coordinates were reduced to two dimensions by combining the non-vertical values (anterior/posterior and medial/lateral measurements) to create a resultant with respect to the shoulder joint. The resultant value was used as the horizontal component input to the shoulder model. The associated shoulder load output was therefore considered to be reflective of a net shoulder moment which provides a simpler single indicator than two axially distinct calculated moments.

\section{$2.3 \quad$ OUTPUTS}

The proposed method provides information on the point-to-point hand movement required by a worker to move from task to task in a work cycle. The point-to-point distances are summed to give a cumulative movement required for the whole work cycle - a surrogate indicator for movement-speed related risk. For the left and right hand positions of each task location identified in the work cycle a shoulder load and a percentage of maximum shoulder load capacity are predicted from the shoulder load regression models. All reported values are easily scaled to reflect a full shift exposure.

\subsection{CASE EXAMPLE}

To demonstrate the feasibility of this method a comparison was conducted based on previous work of Neumann et al. (2002) who studied the ergonomics impact of automation and workstation changes in an electronics company. The original workstations presented parts in a half circle on the workstation surface in front of the assembly operator. Base components were pulled from a cart on one side of the station and completed product was placed to a cart on the other side of the workstation. The new system that was introduced included a conveyor to transport the product. This resulted in the stock being placed behind the conveyor and raised up onto two levels of shelves to avoid contact with the moving product. The hand locations for these workstations have been recreated for this demonstration. Original two-dimensional data was used when available. Location estimations from available 
design information were made when data did not exist. A comparison of the outputs using the method proposed in this paper follows. This comparison was conducted assuming a $50^{\text {th }}$ percentile female using only their right hand to complete all tasks in order to capture the total shoulder loading for the tasks modeled.

\subsection{RESULTS}

\subsection{HAND MOVEMENT}

The hand movement required for the original workstation and the new workstation are shown in Figure 1. The overhead view has two zones of acceptability overlaid on it. The dashed arcs represent the reach distance permissible for repetitive movements whereas the solid arcs reflect the acceptability for occasional reaches. The hand locations of the new design are on the outer boundary and exceeding the guideline for repetitive work however it is less severe than the hand locations required for the new workstation design.
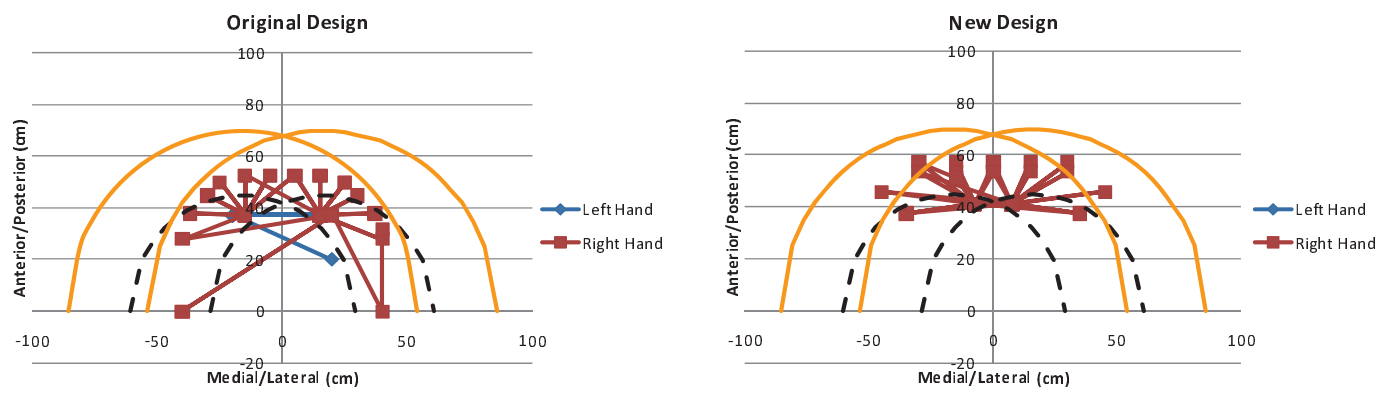

Figure 1. Overhead view of anticipated hand movement required to complete the workstation tasks for the Original Design (left) and New Design (right) as found in Neumann et al. (2002).

The cumulative right hand travel distance for the workstation tasks are shown in Table 2 for the work cycle and the shift. There was a 5.3\% decrease in cumulative hand travel on a per work cycle basis in the new design. Over a full shift the new design increased the required hand travel by $10.0 \%$ due to a cycle time decrease of $13.9 \%$ in the new design.

Table 2. Assembly task shoulder load and cumulative hand travel calculated values for the proposed virtual tool and the observational tool (Obs. Tool) used by Neumann et al. (2002).

\begin{tabular}{|c|c|c|c|c|c|c|c|c|c|}
\hline & \multicolumn{3}{|c|}{ Original Design } & \multicolumn{3}{|c|}{ New Design } & \multicolumn{2}{|c|}{ WS Change (\%) } \\
\hline & & Virtual & Obs. & $\%$ & Virtual & Obs. & $\%$ & Virtual & Obs. \\
\hline & & & & & & & & Tool & \\
\hline \multirow[t]{2}{*}{ Peak } & Resultant & 7.6 & 5.5 & -27.6 & 9.4 & 6.3 & -32.8 & 23.4 & 14.5 \\
\hline & $\%$ MVC & 24.9 & & & 30.9 & & & 23.8 & \\
\hline \multirow[t]{2}{*}{ Average } & Resultant & 5.2 & 3.9 & -21.5 & 5.6 & 4.5 & -20.0 & 7.3 & 13.7 \\
\hline & $\%$ MVC & 16.9 & 11.4 & -32.4 & 19.2 & 14.6 & -19.8 & 7.9 & 28.1 \\
\hline \multicolumn{2}{|c|}{ Hand Travel } & & & & & & & & \\
\hline \multicolumn{2}{|c|}{ Per Work Cycle (m) } & 11.24 & & & 10.64 & & & -5.3 & \\
\hline \multicolumn{2}{|c|}{ Per Shift $(m) *$} & 21.47 & & & 23.62 & & & 10.0 & \\
\hline
\end{tabular}

${ }^{*}$ Full shift assumed to be 7.5 working hours. Cycle time $=141.1 \mathrm{~s} /$ product for Original and 121.5 s/product for New (from Observed cycle time in Table 4, Neumann et al. (2002)).

$\%$ Diff $=100 *$ (Obs. Tool value - Virtual Tool value) / Virtual Tool value

WS (Workstation) Change $\%=100 *$ (New Design - Original Design) $/$ Original Design 


\subsection{SHOULDER LOAD}

Shoulder load increased $(7.3-28.1 \%$ - Table 2$)$ in all measures when switching to the new workstation design. This result was consistent regardless of the assessment method used. Comparison between the virtual tool method proposed and the observational tool of Neumann et al. (2002) showed that the virtual tool predicted higher values in all comparable measures.

\subsection{DISCUSSION}

The comparison with the work of Neumann et al. (2002) demonstrates the potential for the virtual tool to be used without a real world system being present. As in the observational tool of Neumann et al. (2002) the virtual tool predicted the larger shoulder demands in the new design. Magnitudes were much greater though in the virtual tool prediction. Differences between these outcomes could be attributed to the lack of specific, original data for task locations on either side of the midline of the workstation, differences in anthropometrics modeled, or accuracy of the shoulder model used. The work of Neumann et al. (2002) used two-dimensional modelling based on postures observed from video. Hand locations were therefore best estimated for tasks not directly in front of the worker from pictures of layouts and made relative to the workstation origin required for the virtual tool. Closer examination of a task that very closely matched the task from Neumann et al. (2002) did show the virtual tool magnitude to be similar in the new design but had a greater difference in the original workstation. Both of these tasks did have an off plane component to them with the new design being closer to the centre of the worker. Further shoulder load difference is likely due to the shoulder model of the virtual tool being a developed regression model and not from the original source like Neumann et al. (2002). In the virtual tool, off plane tasks combine the horizontal components of the location to make a resultant. Even with small distances from the midline, subtle differences will result in a horizontal resultant component that will be entered into the model. Work is underway to determine the effect of the use of a resultant and the possible benefits of a three dimensional shoulder model. Although the re-creation of these two workstation comparisons attempted to mimic the workstation layout and methods to complete the tasks, the anthropometrics that were modeled in this comparison had slight differences. It is expected that this would result in only small discrepancies in shoulder moment but it could be more pronounced in the percentage of maximum capability depending on the magnitude of the normalizing value.

The method presented aims to develop an inexpensive, simple assessment process that can be used in 'virtual' stages of early system design before a worker can be observed. This allows adjustments to be made to reduce loading while changes are least expensive and solution options are greatest. From task hand location inputs it provides a quick means to visualize hand motion and predict shoulder load. Relative differences between two workstations are easily analyzed as are "What if" scenarios. The concept has further potential to include movement time assessment that can give an understanding of the speed of movement required to complete the tasks and more knowledge about potential worker risks. Further, information about the task time requirements can be coupled with wage rates to strengthen the tool's impact through better business case analysis potential.

A number of simplifying assumptions are made in this virtual tool approach. Calculation of point-to-point task distances, which are used to determine cumulative hand travel, uses a direct straight-line connection between task locations. This therefore represents the minimum 
requirements for hand movement. The shoulder load regression model that is initially used is based on two-dimensional information while the hand location values determined are in three-dimensions. The two horizontal plane values are subsequently combined to form a resultant that is used as the input corresponding to the vertical component. The shoulder value output is therefore considered representative of a net shoulder load.

Like many of the assessment tools available, the method presented is not completely inclusive of all risks faced by a light assembly worker. The virtual tool presented uses the hand location as a means to anticipate shoulder load. Within light assembly work, employees can be sitting or standing and may vary their reach posture over time. Although this method is currently limited to sitting operations it has no consideration to neck or spinal loads placed on the worker. Hand, wrist and finger specific assessments are also not estimated but need to be investigated because of the hand intensive nature of this type of assembly. With near negligible part weights the risk of high force tasks is low however the concern for cumulative loading is significant due to the amount of repetition found in light assembly work. The current method does not yet completely capture the cumulative risk exposure for a worker, although the cumulative hand movement indicator may provide a useful marker for this kind of hazard. An advantage of the low part weight is that the shoulder model can be simplified to be based only on the effect of gravity on the limbs. When coupled with the model being a predictor of net shoulder demand because of the twodimensional inputs it is possible that the accuracy in the output is decreased. The effect of these assumptions and the need for a three-dimensional shoulder model are currently being investigated.

The method demonstration presented here has been developed initially for two groups of the working population $-95^{\text {th }}$ percentile male and $50^{\text {th }}$ percentile female based on data available from earlier shoulder load regression work. In principle any anthropometric could be applied within this technique Expanding the coverage of the working population will capture typical percentiles used for reach and strength capabilities during workstation design and worker suitability assessments as well as a better understanding of the varied work demand exposures faced by the working population.

The origin used to determine the task hand locations allows the potential for future expansion to other anthropometric distributions, potentially other shoulder models as well as the possibility for application to sitting and standing workstations. With this flexibility from the origin used the assumption is made that each of these individuals will sit or stand at a standard distance and orientation with respect to the workstation. Understandably subtle differences in self-selected worker orientation to the workstation and personal techniques may impact the tasks demands that the worker may actually face.

\subsection{FUTURE WORK}

While the tool is currently capable of providing virtual and existing workstation comparisons to support continuous improvement of designs, a risk-calibration study would be required to provide some estimate of the hazard levels associated with a particular level of cumulative shoulder moment or hand travel distance per shift. Future work will involve developing the tool to have an increased application for production planning by incorporating movement time measurement (MTM) estimations. Pilot work has used MTM values to estimate time requirements for the movement distances calculated in the program. Early result summaries have provided a quick view of the interplay between task timing changes and the load exposure for the operator. Adding inputs to describe the type of grasping and placing 
required within the work cycle will allow the tool to provide complete work cycle time estimation. A further extension of the tool to allow an understanding of the speed of movements needed to complete the tasks and, separately, an understanding of potential postures necessary for task vision requirements. An effective method combining all results needs to be investigated since it could potentially help to better prescribe stock placement and work flow allowing the workstation setup to be simultaneously optimized for worker and system benefits. Sources of variability and error in the method must also be further investigated.

\subsection{CONCLUSION}

The tool and process presented demonstrates a concept for a cost effective and simple method to predict operator shoulder demand when designing light assembly workstations. With inputs of hand coordinates obtained from CAD drawings, other design information, or measurement from an actual workstation, for each task in the work cycle, the tool can calculate cumulative hand travel distances (a novel exposure metric) and shoulder moments. The method has been demonstrated to work as both a predictive and comparative tool. Its potential for use in a virtual setting, using design specifications, gives engineers and ergonomists the ability to better identify and manage potential operator risk at the earliest possible design stages when it is cheaper, easier, and faster to make improvements to the layout and task distribution.

\section{ACKNOWLEDGEMENTS}

This work has been supported by the National Science and Engineering Research Council of Canada (NSERC) and by the Workplace Safety and Insurance Board (WSIB) of Ontario.

\section{REFERENCES}

Dul, J., \& Neumann, W.P. (2009). Ergonomics contributions to company strategies. Applied Ergonomics, 40, 745-752.

Miles, B.L., \& Swift, K. (1998). Design for manufacture and assembly. Manufacturing Engineer, 77(5), 221-224.

Neumann, W.P., Kihlberg, S., Medbo, P., Mathiassen, S.E., \& Winkel, J. (2002). A case study evaluating the ergonomic and productivity impacts of partial automation strategies in the electronics industry. International Journal of Production Research, 40(16), 40594075. 